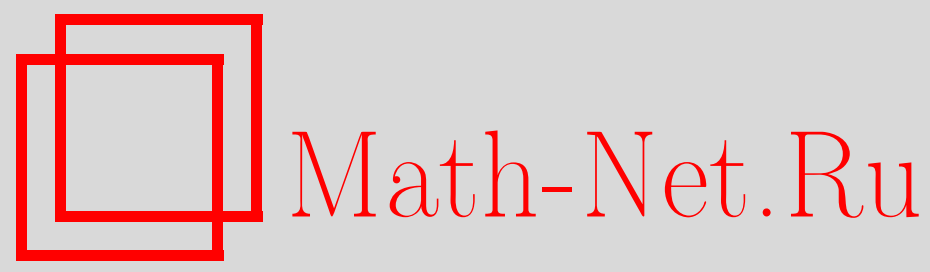

Д. В. Осин, Элементарные классы групп, Матем. заметки, 2002, том 72, выпуск 1, 84-93

DOI: https://doi.org/10.4213/mzm406

Использование Общероссийского математического портала Math-Net.Ru подразумевает, что вы прочитали и согласны с пользовательским соглашением http://www.mathnet.ru/rus/agreement

Параметры загрузки:

IP: 3.80 .253 .173

26 апреля 2023 г., 16:20:49

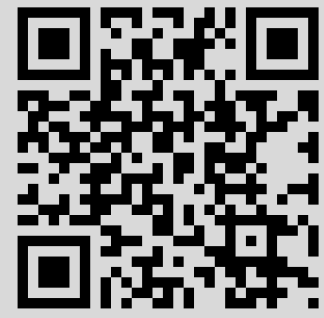




\title{
ЭЛЕМЕНТАРНЫЕ КЛАССЫ ГРУПП
}

\author{
Д. В. Осин
}

Пусть $B$ - некоторый класс групп. Определим элементарный класс с базой $B$ как наименьший к ласс групп, который содержит $B$ и замкнут относительно перехода к подгруппам, факторгруппам, групповым расширениям и направленным пределам. В данной работе изучаются свойства таких классов. Обсуждаются некоторые приложения к теории элементарньх аменабельных групп и связь с классами Куроша-Черникова обобщенных разрешимых групп.

Библиографоия: 13 названий.

1. Введение. В настоящей работе мы рассматриваем некоторые семейства груп, которые могут быть получены естественным образом из заданных. Более точно, пусть $B$ - некоторый класс груп. Определим әлементарный класс с базой $B, \mathscr{E}(B)$, как наименьший класс групп, которьй содержит $B$ и замкнут относительно следующих четырех стандартных операций:

(S) взятие подгрупш;

(Q) взятие фактор груп;

(Е) расширения груп;

(L) взятие направленных (индуктивных) пределов.

Идея рассмотрения элементарных классов возникает в связи с теорией элементарных аменабельных групп, восходящей к классическим работам фон Неймана [1] и Дэя [2]. В наших терминах класс элементарных аменабельных груп $E G$ - это в точности элементарный класс с базой, состоящей из всех абелевых и конечных групп.

Мы получаем описание элементарных классов с произвольной базой, которое обобщает и улучшает данное Чоу описание элементарных аменабельных групा [3]. Используя полученный результат, мы исследуем свойства груп, которые переносятся с базы на весь элементарньй класс. В частности, мы находим достаточное условие для такого переноса и рассматриваем его приложения в ряде случаев. Также изучается связь между элементарными классами и теорией обобщенных разрешимых групп Куроша-Черникова [4], [5].

Основная часть настоящей заметки была написана во время визита автора в Женевский университет; я благодарен П. де ля Арпу за приглашение, а также за постоянное внимание к данной работе. Также я хотел бы поблагодарить Р.И. Григорчука и А. Ю. Ольшанского за полезные замечания и комментарии.

Работа выполнена при поддержке Российского фонда фундаментальных исследований, грант № 99-01-00894, и Швейцарского национального научного фонда. 
2. Основные результаты. Для описания элементарных классов групп нам потребуется следующая вспомогательная конструкция. Пусть $B$ - заданньй базовый класс и класс $\mathscr{E}_{0}(B)$ состоит из одной тривиальной групшы. Предположим, что $\alpha>0$ - некоторьй ординал и что мы уже определили классы $\mathscr{E}_{\beta}(B)$ для всех ординалов $\beta<\alpha$. Тогда положим

$$
\mathscr{E}_{\alpha}(B)=\bigcup_{\beta<\alpha} \mathscr{E}_{\beta}(B),
$$

если $\alpha$ является предельным ординалом. Если же $\alpha$-непредельный ординал, определим $\mathscr{E}_{\alpha}$ как класс всех групп, которые могут быть получены из групп класса $\mathscr{E}_{\alpha-1}$ однократным применением операции $(\mathrm{L})$ или следующей операции.

$\left(\mathrm{E}_{0}\right)$ Для заданной групшы берется ее расширение с помощью грушшы из класса $B$.

Теорема 2.1. Пусть $B$ - некоторый класс групп. Предположсим, что $B$ замкнут относительно операчий (S) $u(\mathrm{Q})$. Тогда

$$
\mathscr{E}_{\alpha}(B)=\bigcup_{\alpha} \mathscr{E}_{\beta}(B)
$$

где оббединение берется по всем ординальным числам.

Следующее описание немедленно вытекает из теоремы 2.1.

СлЕДСТВИЕ 2.1. Элементарный класс $\mathscr{E}(B)$ есть наименьиий класс әрупп, содержащий В и замкнутый относительно $\left(\mathrm{E}_{0}\right)$ u $(\mathrm{L})$.

Пусть теперь $P$ - некоторое свойство, т.е. предикат на классе всех групп. Скажем, что $P$ является $S$-замкнутым (соответственно $Q$-замкнутым, $E$-замкнутыл), если для всякой групшы $G$, обладающей $P$, любая подгруппа $G$ (гомоморфньй образ или расширение с помошью групшы, обладаюшей $P$, соответственно) обладает $P$. Кроме того, мы будем говорить, что $P$ является $L$-замкнутым, если направленный предел $H=\bigcup_{\lambda \in \Lambda} H_{\lambda}$ обладает свойством $P$ всегда, когда каждая из групп $H_{\lambda}$ обладает $P$. Заметим, что любая группа является индуктивным пределом своих конечно порожденных подгрупш. Следовательно, $P L$-замкнуто тогда и только тогда, когда локальная теорема справедлива для $P$ (это означает, что любая группа, все конечно порожденные подгрупшы которой обладают свойством $P$, сама обладает $P$ ). Особенно мы будем интересоваться теми свойствами груп, которые $L$-замкнуты и $E$-замкнуты одновременно (или $L E$-замкнуты, для краткости). Используя следствие 2.1, мы легко получаем следующую теорему.

Tеорема 2.2. Пусть $B-S Q$-замкнутый класс әрупn, $P-L E$-замкнутое свойство групп. Предполохим, что всякая группа из В обладает свойством $Р$. Тогда любая группа из әлементарного класса $\mathscr{E}(B)$ обладает $P$.

ОПРЕДЕЛЕНИЕ 2.1. Будем говорить, что класс груп $\mathscr{C}$ является внешним, если для любого $S Q$-замкнутого класса $B$ из $\mathscr{C} \cap B=\varnothing$ следует $\mathscr{C} \cap \mathscr{E}(B)=\varnothing$.

Например, класс неаменабельных групп является внешним. Другие примеры приводятся в следующей теореме. 
ТЕОРема 2.3. Следующие классы являются внешними:

1) класс конечно порожденных бесконечных периодических групп;

2) класс нетривиальных конечно порождденных делимых групп;

3) класс конечно порохсенных бесконечных простых групп;

4) класс конечно порожденных бесконечных групп, имеющих конечное число классов сопряженности.

Необходимо заметить, что все классы, упомянутые вьше, непусты (интересуюшихся читателей мы отсылаем к книге [6]).

Напомним, что функция роста $\gamma_{G}^{X}: \mathbb{N} \rightarrow \mathbb{N}$ группы $G$, порожденной конечным множеством $X$, определяется формулой

$$
\gamma_{G}^{X}(n)=\operatorname{card}\left\{g \in G:\|g\|_{X} \leqslant n\right\},
$$

где $\|g\|_{X}$ обозначает словарную длину $g$. Показатель әкспоненииального роста $G-$ это число

$$
\omega(G, X)=\lim _{n \rightarrow \infty} \sqrt[n]{\gamma_{X}^{G}(n)}
$$

Вьшеупомянутьй предел существует ввиду субмультипликативности $\gamma_{X}^{G}$.

Говорят, что группа $G$ имеет экспоненциальньй рост (субэкспоненциальньй рост), если $\omega(G, X)>1$ (соответственно $\omega(G, X)=1$ ). Если существуют константы $C, d>0$ такие, что $\gamma_{G}^{X}(n) \leqslant C n^{d}$ для всех $n \in \mathbb{N}$, то говорят, что $G$ имеет полиномиальный рост. Вопрос о существовании конечно порожденной группы промежуточного (т.е. субэкспоненциального, но не полиномиального) роста известен как проблема Милнора. Отрицательный ответ был получен в 60-70-е годы в некоторых частных случаях, но лишњ недавно Григорчуком найдены первые примеры конечно порожденных групп, имеющих промежуточньй рост [7].

Используя теорему 2.2, мы получаем отрицательный ответ на вопрос Милнора для элементарных классов, обобщая тем самым хорошо известные результаты о разрешимых и элементарных аменабельных групшах [3], [8].

ТЕОРема 2.4. Класс конечно порохденных групп промехуточного роста является внешним.

Другое приложение теоремы 2.2 можно найти в п. 4, где мы получаем описание элементарных аменабельных групп с условиями конечности.

ТЕОРема 2.5. 1) Всякая әлементарная аменабельная группа с условием максимальности для подгрупп почти полиииклическая.

2) Всякая әлементарная аменабельная группа с условием минимальности для подәрупп локально конечна.

Наша следующая цель - описать связь между элементарными классами и классами групп Куроша-Черникова. Напомним, что множество подгрупп $\mathfrak{S}=\left\{G_{\mu}\right\}_{\mu \in M}$ группы $G$ образует разрешимый субнормальный ряд, если выполнены следуюшие условия.

1) Множество индексов $M$ упорядочено, т.е. для любых $\nu, \mu \in M$ либо $\nu \leqslant \mu$, либо $\mu \leqslant \nu$.

2) $G_{\mu} \leqslant G_{\nu}$ при $\mu<\nu$.

3) $\mathfrak{S}$ замкнуто относительно любых пересечений и объединений. 
4) Положим $G_{\mu}^{\sharp}=\bigcap_{\nu>\mu} G_{\nu}$. Тогда $G_{\mu}^{\sharp}-$ нормальная подгрупша в $G_{\mu}$ и $G_{\mu} / G_{\mu}^{\sharp}$ абелева при любом $\mu \in M$.

5) $\mathfrak{S}$ содержит одновременно $\{1\}$ и $G$.

Ряд $\mathfrak{S}$ назьвается вполне упорядоченным, если $G_{\mu}^{\sharp} \neq G_{\mu}$ для любого $\mu \in M$.

Говорят, что группа $G$ обладает свойством $S N$, если в $G$ существует разрешимьй субнормальньй ряд. Далее, если $G$ допускает вполне упорядоченньй разрешимый субнормальный ряд, то говорят, что она обладает $S N^{*}$. Мы также обозначаем через $S N$ и $S N^{*}$ классы всех групп, обладающих свойствами $S N$ и $S N^{*}$ соответственно.

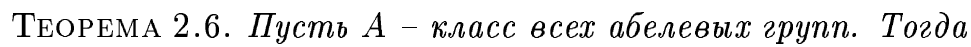

$$
S N^{*} \subset \mathscr{E}(A) \subset S N
$$

В частности, из теоремы 2.6 следует, что любая $S N^{*}$-группа является (элементарной) аменабельной. Это не очевидно априори; так, например, каждая свободная группа обладает свойством $S N$ (как и любая другая групша, апшроксимируемая разрешимыми). Таким образом, включение $S N \subseteq A G$, где $A G$ - класс всех аменабельных групп, не имеет места. Отсюда следует, что включение $\mathscr{E}(A) \subset S N$ является строгим. Более того, в п. 5 будет показано, что и первое включение в (1) строгое. Заметим также, что свойство $S N^{*}$ не эквивалентно разрешимости даже в случае конечно порожденных групп (см. примеры в конце п. 5).

3. Элементарные классы. На протяжении данного пункта мы фиксируем некоторьй $S Q$-замкнутьй класс $B$, обозначаем класс $\mathscr{E}_{\alpha}(B)$ через $\mathscr{E}_{\alpha}$ и $\mathscr{E}(B)$ через $\mathscr{E}$ для краткости. Наша основная цель - доказать теорему 2.1. Начнем со вспомогательных лемм.

ЛЕмма 3.1. Класс $\mathscr{E}_{\alpha}$ является $S Q$-замкнутым для каждого ординала $\alpha$.

ДоКАЗАТЕЛЬСТВО. Применим трансфинитную индукцию по $\alpha$. Лемма очевидна для $\mathscr{E}_{0}$. Предположим, что $\alpha>0$ и что $\mathscr{E}_{\beta}$ является $S Q$-замкнутым, если $\beta<\alpha$. Пусть $G \in \mathscr{E}_{\alpha}, C$ - подгруппа в $G$, и $D$ - образ $G$ при некотором гомоморфизме $\varphi$. Нам надо показать, что $C, D \in \mathscr{E}_{\alpha}$. Если $\alpha-$ предельньй ординал, то $G \in \mathscr{E}_{\beta}$ для некоторого $\beta<\alpha$. Следовательно, $C, D \in \mathscr{E}_{\beta} \subseteq \mathscr{E}_{\alpha}$ по предположению индукции.

Если $\alpha$ - непредельньй ординал, то либо $G$ является расширением вида

$$
1 \rightarrow E \rightarrow G \stackrel{\theta}{\rightarrow} F \rightarrow 1
$$

для некоторых $E \in \mathscr{E}_{\alpha-1}$ и $F \in B$, либо любая конечно порожденная подгруппа в $G$ принадлежит $\mathscr{E}_{\alpha-1}$. В первом случае $C$ изоморфна расширению $C_{1}=C \cap E$ с помощью $C_{2}=\theta(C)$, а $D$ является расширением $D_{1}=\varphi(E)$ с помощью $D_{2}=\varphi(F)$. По предположению $C_{1}, D_{1} \in \mathscr{E}_{\alpha-1}$. Так как $B$ является $S Q$-замкнутым, $C_{2}$ и $D_{2}$ принадлежат $B$. Следовательно, $C, D \in \mathscr{E}_{\alpha}$. Во втором случае заметим, что всякая конечно порожденная подгруппа в $C$ и в $D$ принадлежит $\mathscr{E}_{\alpha}$, будучи подгруппой или факторгруппой конечно порожденной подгрупшы в $G$. Значит, снова $C, D \in \mathscr{E}_{\alpha}$.

Лемма 3.2. Пусть группа $G$ является расширением группы $F \in \mathscr{E}_{\alpha}$ при помощи группы $H \in \mathscr{E}_{\beta}$ для некоторых ординалов $\alpha$ и $\beta$. Тогда существует ординал $\gamma$ mакой, что $G \in \mathscr{E}_{\gamma}$. 
ДоКАЗАТЕЛЬСТВо. Применим трансфинитную индукцию по $\beta$. Случай $\beta=0$ тривиален. Предположим, что $\beta>0$ и мы доказали лемму для всех ординалов $\delta<\beta$. Если $\beta$ - предельный ординал, то $H \in \mathscr{E}_{\varepsilon}$ для некоторого $\varepsilon<\beta$ и требуемое $\gamma$ существует по предположению индукции. Теперь пусть $\beta$ - непредельный ординал. Существуют две возможности получения $H$ из групп класса $\mathscr{E}_{\beta-1}$.

Сначала предположим, что $H$ является расширением $D$ при помощи $E$, где $D \in \mathscr{E}_{\beta}-1$ и $E \in B$. Рассмотрим полный прообраз $D$ при естественном гомоморфизме $G \rightarrow H$ и обозначим его $D_{0}$. Ясно, что $D_{0}$ - расширение $F$ с помощью $D$ и, таким образом, $D_{0} \in \mathscr{E}_{\xi}$ для некоторого $\xi$ по предположению индукции. Следовательно, $G \in \mathscr{E}_{\xi+1}$, так как $G / D_{0} \cong H / D \in B$.

Далее, пусть $H$ - направленньй предел семейства групп $\left\{H_{\lambda}\right\}_{\lambda \in \Lambda}$ и вложений $H_{\lambda} \cup H_{\mu}$ $\subseteq H-\nu$. Рассмотрим полные прообразы $H_{\lambda}^{0}$ групा $H_{\lambda}$ в $G$. По нашему предположению $H_{\lambda} \in \mathscr{E}_{\beta-1}$ для всех $\lambda \in \Lambda$. Значит, $H_{\lambda}^{0} \in \mathscr{E}_{\xi_{\lambda}}$ для некоторых $\xi_{\lambda}$ по предположению индукции. Возьмем сумму $\xi=\sum_{\lambda \in \Lambda} \xi_{\lambda}$. Как известно, $\xi$ - ординал, не меньший, чем любой из $\xi_{\lambda}$. Это влечет $\mathscr{E}_{\xi_{\lambda}} \subset \mathscr{E}_{\xi}$ для всех $\lambda \in \Lambda$ и, таким образом, $H_{\lambda}^{0} \in \mathscr{E}_{\xi}$ для всех $\lambda$. Заметим, что вложения $H_{\lambda} \rightarrow H_{\mu}$ могут быть расширены до вложений $H_{\lambda}^{0} \rightarrow H_{\mu}^{0}$ естественным образом; тогда соответствуюший направленный предел $G_{0}=\bigcup_{\lambda \in \Lambda} H_{\lambda}$ будет изоморфен $G$. Отсюда следует, что $G \in \mathscr{E}_{\xi+1}$.

Теорема 2.1 немедленно следует из леммы 3.1 и леммы 3.2 .

4. Свойства элементарных классов. Продемонстрируем примеры конструирования $L E$-замкнутых свойств групा.

Лемма 4.1. Пусть $P_{1}$ - некоторое $S Q$-замкнутое, а $P_{2}$ - некоторое $L E$-замкнутое свойство групп. Тогда свойство "если группа обладает $P_{1}$, то она обладает $P_{2} "\left(P_{1} \Longrightarrow P_{2}\right.$ для краткости) является $L E$-замкнутым.

ДокаЗАТЕльСтво. Сначала докажем, что $P_{1} \Longrightarrow P_{2}$ является $L$-замкнутым. Предположим, что любая конечно порожденная подгруппа группы $G$ обладает $P_{1} \Longrightarrow P_{2}$. Если $G$ не обладает свойством $P_{1}$, то импликация $P_{1} \Longrightarrow P_{2}$ истинна. Если же $G$ обладает $P_{1}$, то любая ее подгрупша обладает $P_{1}$, так как $P_{1}$ является $S$-замкнутым. Следовательно, любая конечно порожденная подгруппа в $G$ обладает $P_{2}$ по нашему предположению. Принимая во внимание, что $P_{2}$ является $L$-замкнутым, заключаем, что $G$ обладает $P_{2}$ и, таким образом, обладает $P_{1} \Longrightarrow P_{2}$.

Теперь предположим, что имеется точная последовательность

$$
1 \rightarrow M \rightarrow G \rightarrow N \rightarrow 1,
$$

где $M$ и $N$ обладают $P_{1} \Longrightarrow P_{2}$. Если $G$ не обладает $P_{1}$, то импликация $P_{1} \Longrightarrow P_{2}$ справедлива, как и выше. Предположим, что $G$ обладает $P_{1}$. Так как $P_{1}$ является $S Q$-замкнутым, $M$ и $N$ обладают $P_{1}$. Следовательно, $M$ и $N$ обладают $P_{2}$. Значит, $G$ обладает $P_{2}$, так как $P_{2}$ является $E$-замкнутьм.

ПримеР 4.1. Рассмотрим следующие свойства:

$P_{1}$. периодичность;

$P_{2}$. локальная конечность. 
Нетрудно видеть, что $P_{1}$ и $P_{2}$ удовлетворяют условиям предыдущей леммы $(E-$-замкнутость $P_{2}$ следует из хорошо известной теоремы Шмидта [9, теорема 22.3.1]). Следовательно, $P_{1} \Longrightarrow P_{2}$ является $L E$-замкнутьм. Это означает, что если всякая периодическая групш локально конечна в базе $B$, то то же самое справедливо для любой группы из $\mathscr{E}(B)$. Таким образом, мы обобщили хорошо известньй факт, касающийся элементарных аменабельных групп, и доказали первое утверждение теоремы 2.3.

\section{ПРИмеР 4.2. Рассмотрим также свойство}

$P_{3}$. конечность ранга (напомним, что группа $G$ имеет конечньй ранг $d$, если любая конечно порожденная подгруппа в $G$ может быть порождена не более чем $d$ элементами).

Ясно, что $P_{3}$ является $S Q$-замкнутым. Следовательно, свойство $P_{1} \& P_{3}$ является $S Q$-замкнутым, а свойство $\left(P_{1} \& P_{3}\right) \Longrightarrow P_{2}-L E$-замкнутьп. Заметим, что существуют группы, не обладающие $\left(P_{1} \& P_{3}\right) \Longrightarrow P_{2}$ (например, монстры Тарского, см. [6, гл. 9]; эти группы 2-порождены, бесконечны, конечной экспоненты и таковы, что любая их собственная подгруппа циклична). Таким образом, мы показали, что если группы типа монстров Тарского отсутствуют в базе $B$, то они не появляются и в $\mathscr{E}(B)$.

Свойство $\left(P_{1} \& P_{3}\right) \Longrightarrow P_{2}$ может быть интересно, скажем, для элементарных классов, которые не обладают $P_{1} \Longrightarrow P_{2}$, т.е. содержат конечно порожденные бесконечные периодические группы. Пример такого класса может быть построен следующим образом. Пусть $B_{0}=\{G\}$, где $G-2$-группа Григорчука (см. [7]). Обозначим через $B S Q$-замыкание $B_{0}$. Заметим, что любая собственная факторгруппа $G$ конечна, так же, как и любая подгруппа конечного ранга в $G$ (как известно [10], любая конечно порожденная подгрупша групшы Григорчука соизмерима с прямым произведением конечного числа копий $G$ ). Отсюда следует, что любая групша из $\mathscr{E}(B)$ удовлетворяет $\left(P_{1} \& P_{3}\right) \Longrightarrow P_{2}$.

ДОКАЗАТЕЛЬСТВО ТЕОРЕМЫ 2.2. Трансфинитной индукций по $\alpha$ мы покажем, что свойством $P$ обладает каждая группа $G \in \mathscr{E}_{\alpha}$. Случай $\alpha=0$ тривиален. Предположим теперь, что $\alpha>0$. Если $\alpha-$ предельньй ординал, то $G \in \mathscr{E}_{\beta}$ для некоторого $\beta<\alpha$ и, таким образом, $G$ обладает $P$ по предположению индукции. Если же ординал $\alpha$ непредельный, то из $L E$-замкнутости $P$ следует, что $G$ обладает $P$. Это завершает доказательство ввиду теоремы 2.1 .

ДокАЗАТЕЛЬСТво ТЕОРЕмЫ 2.3. Первое утверждение теоремы уже доказано в примере 4.1. Остается показать, что следующие свойства являются $L E$-замкнутьми (мы записьваем их в сокращенных обозначениях):

$P_{1} .(($ делимость $) \&($ конечная порожденность $)) \Longrightarrow$ (тривиальность);

$P_{2}$. ((простота) \& (конечная порожденность $\left.)\right) \Longrightarrow$ (конечность);

$P_{3}$. ((существование лишь конечного числа классов сопряженности) \& (конечная порожденность $)) \Longrightarrow$ (конечность).

Заметим, что добавление требования конечной порожденности в посылку импликаций делает свойства $P_{1}, P_{2}, P_{3} L$-замкнутыми. Таким образом, достаточно проверить $E$-замкнутость. Пусть некоторая групша $G$ есть расширение $F$ при помощи $H$.

Сначала предположим, что $F$ и $H$ обладают $P_{1}$. Если $G$ не является делимой или конечно порожденной, то $P_{1}$ истинно. Теперь заметим, что всякая факторгруппа конечно порожденной делимой группы конечно порождена и делима. Следовательно, $H$ 
тривиальна по предположению индукции. Это означает, что $G$ совпадает с $F$ и, следовательно, обладает $P_{1}$. Второе утверждение теоремы доказано.

Теперь, предполагая, что $F$ и $H$ обладают $P_{2}$, докажем, что и $G$ обладает $P_{2}$. Если $G$ не является простой, то $P_{2}$ истинно. Если $G$ проста, мы имеем $F=1$ или $F=G$. Группа $G$ обладает $P_{2}$ в обоих случаях по предположению индукции.

Наконец, предположим, что $F$ и $H$ обладают $P_{3}$. Как и выше, мы можем считать $G$ конечно порожденной и имеющей лиш конечное число классов сопряженных элементов. Очевидно, эти свойства переносятся на $H$. Значит, группа $H$ конечна, а $F$ конечно порождена, так как она имеет конечный индекс в $G$. Более того, $F$ имеет конечное число классов сопряженности по той же причине. Из наших предположений следует, что $F$ конечна, а значит, такова и $G$.

Напомним, что группа назьвается почти полициклической, если она содержит полициклическую подгруппу конечного индекса.

Лемма 4.2. Пусть $G$ - расширение конечной группы $K$ при помощи полициклической әруппы $H$. Тогда $G$ почти поличиклическая.

ДоКАЗАТЕЛЬСТво. Положим $m=|K|$. Тогда для любого $g \in G$ действие элемента $g^{m !}$ на $K$ сопряжениями тривиально, так как орбита действия $\langle g\rangle$ содержит не более $m$ элементов. Рассмотрим подгруппу $G_{0}=\left\langle g^{m !}: g \in G\right\rangle$ и положим $K_{0}=G_{0} \cap K$. Ясно, что $K_{0}$ центральна в $G$ (в частности, $K$ конечная абелева). Далее, имеем

$$
G_{0} / K_{0} \cong G_{0} K / K \leqslant H \text {. }
$$

Это влечет полицикличность $G_{0}$. Наконец, мы имеем точную последовательность

$$
1 \rightarrow K / K_{0} \cong G_{0} K / G_{0} \rightarrow G / G_{0} \rightarrow G / G_{0} K \rightarrow 1
$$

где $K / K_{0}$ и $G / G_{0} K$ являются конечными, будучи факторгруппой конечной грушы и разрешимой периодической группой соответственно. Следовательно, индекс $\left[G: G_{0}\right]$ конечен.

ЛЕмма 4.3. Пусть $G$ - расиирение вида

$$
1 \rightarrow F \rightarrow G \stackrel{\varphi}{\rightarrow} P \rightarrow 1
$$

әде F и $P$ - почти полициклические группь. Тогда $G$ почти поличиклична.

ДокАЗАТЕльСтво. Обозначим через $P_{0}$ и $F_{0}$ нормальные полициклические подгруппы конечного индекса в $P$ и $F$ соответственно и рассмотрим $G_{0}=\varphi^{-1}\left(P_{0}\right)$. Пусть $\left|F / F_{0}\right|=i$. Тогда подгруппа $F^{i}=\left\langle f^{i}: f \in F\right\rangle$ лежит в $F_{0}$ и, таким образом, является полициклической. Более того, $F^{i}$ - характеристическая подгруппа в $F$. Следовательно, $F^{i}$ - нормальная подгруппа в $G_{0}$. Очевидно, что факторгруппа $G_{0} / F^{i}$ есть расширение конечной группы при помощи полициклической. По лемме 4.2 существует полициклическая подгруппа $Q$ конечного индекса в $G_{0} / F^{i}$. Обозначим через $L$ полный прообраз $Q$ при естественном гомоморфизме $G_{0} \rightarrow G_{0} / F^{i}$. Тогда $L-$ расширение $F^{i}$ при помощи $Q$ и, таким образом, $L$ полициклична. Легко видеть, что индекс $[G: L]$ конечен.

Нам необходим также следующий результат, принадлежащий Россету. 
Лемма 4.4 (Россет, [11]). Пусть $G$ - конечно порожденное расширение некоторой группы $F$ с помощью почти разрешимой группы. Если $F$ не является конечно порожсденой, то $G$ имеет әкспоненциальный рост.

Теперь мы можем приступить к доказательству теоремы 2.4 .

ДокАЗАТЕЛЬСТво ТЕОРЕмЫ 2.4. Достаточно доказать $E$-замкнутость свойства

$M$. (конечная порожденность) $\Longrightarrow$ (либо полиномиальность, либо экспоненщиальность роста).

Допустим, мы имеем точную последовательность

$$
1 \rightarrow F \rightarrow G \rightarrow H \rightarrow 1
$$

где $F$ и $H$ обладают $M$. Очевидно, мы можем считать, что $G$ конечно порождена. Тогда $H$ тоже конечно порождена и имеет либо полиномиальньй, либо экспоненциальньй рост. Если $H$ - групша экспоненциального роста, то такова и $G$. Таким образом, мы можем считать, что рост $H$ полиномиален, т.е. $H$ почти нильпотентна по теореме Громова [12]. Если $F$ не конечно порождена, $G$ имеет экспоненциальньй рост по лемме 4.4. Далее, если $F$ конечно порождена, то она является группой либо экспоненциального, либо полиномиального роста. Во втором случае $G$ снова имеет экспоненциальный рост. Наконец, если $F$ - группа полиномиального роста, т.е. $F$ почти нильпотентна, то $G$ почти разрешима по лемме 4.3. Значит, $G$ имеет либо полиномиальньй, либо экспоненциальный рост [8].

ДоКАЗАТЕЛЬСТво тЕОРЕмы 2.5. 1) Рассмотрим следующие свойства:

$R_{1}$. условие максимальности для подгрупп;

$R_{2}$. почти полищикличность.

Заметим, что $R_{2}$ является $E$-замкнутым по лемме 4.3 , но не является $L$-замкнутым. Однако легко проверить, что свойство $R_{1} \Longrightarrow R_{2} L$-замкнуто, так как группа с условием максимальности для подгрупп должна быть конечно порождена. Кроме того, поскольку $R_{1} S Q$-замкнуто, мы заключаем, что $R_{1} \Longrightarrow R_{2}$ является $E$-замкнутым. Действительно, пусть некоторая группа $G$ есть расширение $F$ при помощи $H$, а $F$ и $H$ обе обладают $R_{1} \Longrightarrow R_{2}$. Если $R_{1}$ ложно для $G$, то $R_{1} \Longrightarrow R_{2}$ истинно. Если $R_{1}$ истинно, то $F$ и $H$ обладают $R_{1}$ ввиду $S Q$-замкнутости $R_{1}$, а следовательно, $F$ и $H$ обладают $R_{2}$. Так как $R_{2} E$-замкнуто, то $G$ обладает $R_{2}$ и импликация $R_{1} \Rightarrow R_{2}$ опять справедлива. Для доказательства теоремы остается заметить, что всякая абелева группа с условием максимальности для подгрупп является полициклической и применить теорему 2.2.

$2)$ Если груша $G$ удовлетворяет условию минимальности для подгрупп, то она периодична. Остается сослаться на первое утверждением теоремы 2.3.

5. Связь с классами Куроша-Черникова. Рассмотрим некоторую группу $G \in$ $S N^{*}$. Если $\mathfrak{S}$ - вполне упорядоченная разрешимая субнормальная система в $G$, обозначим через $\tau(\mathfrak{S})$ ее порядковьй тип и определим $\min G$ формулой $\tau(G)=\min \tau(\mathfrak{S})$, где минимум берется по всем вполне упорядоченным разрешимым системам в $G$. Существование минимального элемента следует из вполне упорядоченности любого множества ординальных чисел. 
ДокАЗАТЕЛЬСтво ТЕоремы 2.6. Прежде всего докажем, что $S N^{*} \subset \mathscr{E}(A)$. Обозначим через $S N_{\theta}^{*}$ подкласс $S N^{*}$, состоящий из всех групп $G$ с условием $\tau(G) \leqslant \theta$. Доказательство будет проводиться индукцией по $\theta$.

Заметим, что $\theta$ не меньше 2 для любой группы $G$, так как всякий субнормальньй ряд должен содержать $G$ и $\{1\}$. Случай $\theta=2$ тривиален. Действительно, $S N_{2}^{*}$ состоит только из абелевых групп. Пусть теперь $\theta>2$; допустим, что мы уже доказали вхождение $S N_{\pi}^{*} \subset \mathscr{E}(A)$ для всех $\pi<\theta$. Пусть $G$-групша из $S N_{\theta}^{*}$. Заметим, что $G$ является направленным пределом своих подгрупп, образующих субнормальньй ряд, каждая из которых имеет меньший тип и, следовательно, лежит в $\mathscr{E}(A)$ по предположению индукции. Так как $\mathscr{E}(A)$ является $L$-замкнутым, мы получаем $G \in \mathscr{E}(A)$.

Для того чтобы доказать, что $\mathscr{E}(A) \subset S N$, достаточно заметить, что свойство $S N$ $E$-замкнуто по очевидным причинам и $L$-замкнуто ввиду глубокого результата Куроша и Черникова, передоказанного Мальцевьм (см. [9, теорема 22.3.2]). Таким образом, $\mathscr{E}(A) \subset S N$ по теореме 2.2 .

ПРИмеР 5.1. Чтобы показать, что $S N^{*} \neq \mathscr{E}(A)$, рассмотрим групш, строящуюся следуюшим образом. Положим $Z_{0}=\mathbb{Z}$ и $Z_{i+1}=\mathbb{Z} \imath Z_{i}$. Затем возьмем индуктивный предел $Z_{\omega}$ семейства груп $Z_{i}$ с естественными вложениями $Z_{i} \rightarrow Z_{i+1}$. Ясно, что $Z_{\omega} \in \mathscr{E}(A)$. Мы собираемся доказать, что $Z_{\omega} \notin S N^{*}$.

Пусть $\mathfrak{D}=\left\{D_{\lambda}\right\}_{\lambda \in \Lambda}-$ вполне упорядоченный субнормальный ряд в $Z_{\omega}$. Так как $\mathfrak{D}$ вполне упорядочен, множество индексов $\Lambda$ также вполне упорядочено. Рассмотрим $A=\{1\}^{\sharp}$. По определению $A \neq\{1\}$. Пусть $A \cap Z_{i} \neq\{1\}$ для некоторого $i$. Мы хотим показать, что $D_{\lambda} \leqslant Z_{i}$ для любого $\lambda \in \Lambda$. Это повлекло бы $Z_{\omega} \leqslant Z_{i}$ и привело бы к противоречию.

Применим трансфинитную индукцию по $\lambda$. Обозначим через $\lambda_{0}$ минимальньй элемент множества $\Lambda$. Если $\lambda=\lambda_{0}$, то $D_{\lambda}=1$ и наше утверждение справедливо. Допустим, что $\lambda>\lambda_{0}$ и что мы уже доказали включение $D_{\mu} \leqslant Z_{i}$ для всех $\mu<\lambda$. Положим

$$
D_{\lambda}^{b}=\bigcup_{\mu<\lambda} D_{\mu} .
$$

По определению субнормального ряда $D_{\lambda}^{b} \in \mathfrak{D}$. Если $D_{\lambda}^{b}=D_{\lambda}$, то $D_{\lambda} \leqslant Z_{i}$, так как каждая из групп $D_{\mu}$ лежит в $Z_{i}$ по предположению индукции. Предположим теперь, что $D_{\lambda}^{b} \neq D_{\lambda}$. Тогда, очевидно, $D_{\lambda}=\left(D_{\lambda}^{b}\right)^{\sharp}$. Пусть $D_{\lambda}$ содержит элемент $z \in Z_{k} \backslash Z_{i}$ для некоторого $k>i$. Без ограничения общности мы можем предполагать, что $z \notin Z_{k-1}$. В частности, это означает, что $z$ обладает разложением вида $z=z_{1} z_{2}$ с нетривиальным $z_{2}$, где $z_{1} \in Z_{k-1}$ а $z_{2}$ принадлежит базе сплетения $\mathbb{Z} \backslash Z_{k-1}$. Далее, рассмотрим произвольный нетривиальньй элемент $d \in D_{\lambda}$ такой, что $d \in Z_{i}$. Такой элемент сушествует, так как $A \leqslant D_{\lambda}$ для всех $\lambda>\lambda_{0}$ по выбору $A$. Имеем

$$
[d, z]=d^{-1} z_{2}^{-1} z_{1}^{-1} d z_{1} z_{2}=\left[d, z_{1}\right]\left(z_{2}^{-1}\right)^{z_{1}^{-1} d z_{1}} z_{2}
$$

Так как $z_{1}^{-1} d z_{1} \neq 1$, мы имеем $\left(z_{2}^{-1}\right)^{z_{1}^{-1} d z_{1}} z_{2} \neq 1$. Поскольку $\left(z_{2}^{-1}\right)^{z_{1}^{-1} d z_{1}} z_{2}$ принадлежит базе сплетения $\mathbb{Z} \imath Z_{k-1}$, то $[d, z] \notin D_{\lambda}^{b}$. Это означает, что коммутатор $[d, z]$ имеет нетривиальньй образ в $D_{\lambda} / D_{\lambda}^{b}$, что противоречит предположению об абелевости $D_{\lambda} / D_{\lambda}^{b}$. 
ПРимеР 5.2. Построим пример конечно порожденной $S N^{*}$-группы, которая не является разрешимой. Для этого возьмем любое неразрешимое локально нильпотентное многообразие $\mathscr{V}$ (скажем, многообразие Кострикина $\mathscr{K}_{p}$ для простого $p \geqslant 5$ ). Затем рассмотрим свободную 2-порожденную группу $F$ в многообразии $\mathscr{V} \mathscr{A}$, где $\mathscr{A}$ - многообразие всех абелевых групп. Заметим, что коммутант $[F, F]$ локально нильпотентен и, таким образом, является индуктивным пределом нильпотентных групп. Напомним, что всякая подгруппа нильпотентной группы субнормальна, т.е. может быть включена в некоторьй субнормальньй ряд. Нетрудно видеть, что группа $F$ лежит в $S N^{*}$ (на самом деле, она допускает вполне упорядоченный субнормальньй ряд порядкового типа $\omega+1)$, но $F$ не является разрешимой, так как $[F, F]-\mathscr{V}$-свободная группа счетного ранга. Все отмеченные здесь факты должны быть вполне очевидными для читателя, хорошо знакомогос теорией многообразий групा. Мы не объясняем подробно всех деталей и отсылаем заинтересованных читателей к книгам [6] и [13].

\section{СПИСОК ЦИТИРОВАННОЙ ЛИТЕРАТУРЫ}

[1] von Neumann J. Zur allgemeinen Theorie des Masses // Fund. Math. 1929. V. 13. P. 73-116.

[2] Day M. M. Amenable semigroups // Illinois J. Math. 1957. V. 1. P. 509-544.

[3] Chou Ch. Elementary amenable groups // Illinois J. Math. 1980. V. 24. P. 396-407.

[4] Черников С.Н., Курош А. Г. Разрешимые и нильпотентные группы // УМН. 1947. T. 2. C. $18-59$.

[5] Razmyslov Yu. P. Identities of Algebras and their Representations. Transl. Math. Monographs. V. 138. Providence, R.I.: Amer. Math. Soc, 1994.

[6] Ольшанский А. Ю. Геометрия определяющих соотношений в группах. М.: Наука, 1989.

[7] Григорчук Р.И. К проблеме Милнора о групповом росте // Докл. АН СССР. 1983. T. 271 . C. $30-33$.

[8] Wolf J.A. Growth of finitely generated solvable groups and curvature of Riemannian manifolds // J. Diff. Geom. 1968. V. 2. P. 421-446.

[9] Каргаполов М.И., Мерзляков Ю. И. Основы теории групп. М.: Наука, 1982.

[10] Grigorchuk R. I., Wilson J. Rigidity Properties of Branch Groups. Preprint: University of Geneva, 2001.

[11] Rosset S. A property of groups of non-exponential growth // Proc. Amer. Math. Soc. 1976. V. 54. P. 24-26.

[12] Gromov M. Groups of polynomial growth and expanding maps // Publ. Math. IHES. 1981. V. 53. P. 53-73.

[13] Нейман Х. Многообразия групп. М.: Мир, 1969.

Академия бюджета и казначейства при $\mathrm{M} \Phi \mathrm{P} \Phi$

E-mail: denis.osin@mtu-net.ru 